\title{
OBSTRUCTIONS TO EXTENDING DIFFEOMORPHISMS
}

\section{JAMES MUNKRES ${ }^{1}$}

In [2], we defined a theory of obstructions to constructing a diffeomorphism between two combinatorially equivalent differentiable manifolds. In the present paper, we apply this theory to the problem of extending a diffeomorphism of the boundaries of two manifolds to a diffeomorphism of the manifolds. A corollary is a theorem of $\mathrm{R}$. Thom concerning the equivalence of differentiable structures.

All differentiable manifolds and maps are assumed to be of class $C^{\infty}$; a combinatorial equivalence between two differentiable manifolds is an isomorphism between smooth $\left(C^{\infty}\right)$ triangulations of them.

THEOREM 1. Let $M$ and $N$ be combinatorially equivalent differentiable n-manifolds. Let $\mathrm{Bd} M$ be the disjoint union of $M_{0}$ and $M_{1}$, where each is a union of components of $\mathrm{Bd} M$; similarly, let $\operatorname{Bd} N=N_{0} \cup N_{1}$. Let $f: M_{0} \rightarrow N_{0}$ be a diffeomorphism which is extendable to a combinatorial equivalence $F: M \rightarrow N$. The obstructions to extending $f$ to a diffeomorphism of $M$ onto $N$ are elements of $\mathfrak{F}_{m}\left(M, M_{1} ; \Gamma^{n-m}\right)$; if these obstructions vanish, $f$ may be so extended.

(Here $\mathcal{H}_{m}$ denotes homology based on infinite chains; $m$ ranges from 0 to $n ; M_{1}$ may be empty. The coefficient group $\Gamma^{n-m}$ is defined in [2]; the coefficients are twisted if $M$ is nonorientable.)

Proof. We assume, without loss of generality, that the restriction of $F$ to some neighborhood of $M_{0}$ is a diffeomorphism. (One may justify this as follows: Consider the manifold obtained from $M$ and $M_{0} \times I$ by identifying $x \in M_{0}$ with $(x, 0) \in M_{0} \times I$; it is diffeomorphic to $M$. Obtain a manifold from $N$ and $N_{0} \times I$ similarly. The map which equals $F$ on $M$ and equals the trivial extension of $f$ on $M_{0} \times I$ is a combinatorial equivalence between these manifolds, and its restriction to $M_{0} \times(0,1]$ is a diffeomorphism.)

Restrict $F$ to the manifold $M^{\prime}=M-M_{0}$; it will be a combinatorial equivalence between this manifold and $N^{\prime}=N-N_{0}$. We apply our obstruction theory (in particular, 5.7 of [2]) to the problem of smoothing this map; our object is to obtain a diffeomorphism of $M^{\prime}$ onto $N^{\prime}$ which equals $F$ in a neighborhood of $M_{0}$. Such a diffeomorphism will give the required extension of $f$.

Now $F: M^{\prime} \rightarrow N^{\prime}$ is a diffeomorphism mod the $(n-1)$-skeleton of $M^{\prime}$; we denote it by $F_{n-1}$. As an induction hypothesis, assume $F_{m}$ is a

Received by the editors February 2, 1963.

1 This work was supported by the U. S. Army Research Office (Durham). 
diffeomorphism mod the $m$-skeleton of $M^{\prime}$, which equals $F$ in a neighborhood of $M_{0}$. Let us suppose for the moment that the obstruction $\lambda_{m} F_{m}$ is homologous to zero, $\bmod \mathrm{Bd} M^{\prime}=M_{1}$; let $c$ be the chain it bounds. We may modify $F_{m}$ near the carrier of $c$, obtaining a diffeomorphism $\bmod m, F_{m}^{\prime}: M^{\prime} \rightarrow N^{\prime}$, such that $\lambda_{m} F_{m}^{\prime}=0$. Then $F_{m}^{\prime}$ may be smoothed to $F_{m-1}$, a diffeomorphism $\bmod m-1$. Now the carrier of $\lambda_{m} F_{m}$ is disjoint from some neighborhood of $M_{0}$, because $F_{m}$ is already a diffeomorphism in some such neighborhood. If the carrier of $c$ has the same property, then $F_{m}^{\prime}=F_{m}=F$ in some such neighborhood. It follows that $F_{m-1}$ may be chosen equal to $F_{m}^{\prime}$ in some such neighborhood, since $F_{m}^{\prime}$ is already differentiable there. Providing these suppositions hold at each stage of the induction, the smoothing process can be continued until one obtains a diffeomorphism which equals $F$ in a neighborhood of $M_{0}$.

From the preceding analysis, it is clear that the homology class of our obstruction lies in those homology groups of $M^{\prime}, \bmod M_{1}$, which are based on chains whose carriers have no point of $M_{0}$ as a limit point.(The chains may be infinite, however.) These groups are easily proved to be isomorphic with the groups $\mathcal{F}_{m}\left(M, M_{1} ; \Gamma^{n-m}\right)$; the isomorphism is in fact induced by the inclusion of $M^{\prime}$ into $M$.

TheоRем 2 (Тном [3]). Let $M$ be a differentiable manifold whose boundary has two components $M_{0}$ and $M_{1}$; suppose $M$ is combinatorially equivalent to the differentiable manifold $P \times I$. If $M_{0}$ is diffeomorphic to $P$, so is $M_{1}$, and $M$ is diffeomorphic to $P \times I$.

Proof. Let $f: M_{0} \rightarrow P \times 0$ be a diffeomorphism. If we can extend $f$ to a combinatorial equivalence of $M$ onto $P \times I$, then the theorem follows, for all the obstructions lie in $\mathcal{H}_{m}\left(M, M_{1} ; \Gamma^{n-m}\right)$, and these groups vanish because $M_{1}$ is a deformation retract of $M$. Let $K$ be a complex which serves to triangulate $P$ smoothly. Then any simplicial subdivision of the cell-complex $K \times I$ serves to triangulate $P \times I$ smoothly. Because $M$ is combinatorially equivalent to $P$, any two smooth triangulations of $M$ and $P \times I$ have isomorphic subdivisions ([4]; see also [1]). In particular, there is a smooth triangulation $h: L \rightarrow M$ such that $L$ is a simplicial subdivision of $K \times I$. Assume $h$ carries $|K \times 0|$ onto $M_{0}$, for convenience.

Let $K^{\prime} \times 0$ be the subdivision of $K \times 0$ induced by $L$. Then $K^{\prime} \times I$ is a cell-subdivision of $K \times I$, as is $L$; they have a common simplicial subdivision $L^{\prime}$.

$$
\begin{gathered}
L^{\prime} \stackrel{h}{\stackrel{h}{h}} M, \stackrel{f}{\rightarrow} P . \\
K^{\prime} \times 0 \stackrel{\rightarrow}{\rightarrow} M_{0} \stackrel{\rightarrow}{\rightarrow} P .
\end{gathered}
$$


Let $h_{0}$ be the restriction of $h$ to $|K \times 0|$, then $f h_{0}: K^{\prime} \times 0 \rightarrow P$ is a smooth triangulation. Let $F:|K \times I| \rightarrow P \times I$ be the trivial extension of $f h_{0}$; because $L^{\prime}$ is a subdivision of $K^{\prime} \times I, F$ will be smooth on each simplex of $L^{\prime}$. Hence $F h^{-1}: M \rightarrow P \times I$ is a combinatorial equivalence; its restriction to $M_{0}$ is $f$, as desired.

\section{REFERENCES}

1. J. R. Munkres, Elementary differential topology, Annals of Mathematics Studies No. 54, Princeton Univ. Press, Princeton, N. J., 1963.

2. - Obstructions to the smoothing of piecewise-differentiable homeomorphisms, Ann. of Math. (2) 72 (1960), 521-554.

3. R. Thom, Des variêtés triangulées aux varietés différentiables, Proc. Internat. Congr. Math., 1958, pp. 248-255, Cambridge Univ. Press, Cambridge, 1960.

4. J. H. C. Whitehead, On C1-complexes, Ann. of Math. (2) 41 (1940), 809-824.

Massachusetts Institute of Technology 